\title{
Os meios alternativos de comunicação como ferramenta para a prevenção do câncer de próstata
}

\author{
Alternative means of communication as a tool for preventing prostate cancer \\ Los medios alternativos de comunicación como herramienta para la prevención del cáncer \\ de próstata
}

Bruna Lima'; Elizabeth Rose da Costa Martins ${ }^{I I}$; Raquel Conceição de Almeida Ramos ${ }^{\text {III }}$; Cristiane Maria Amorim Costa ${ }^{I V}$; Araci Carmen Clos ${ }^{V}$; Jaqueline Inácio Correia Ferreira ${ }^{V I}$

\begin{abstract}
RESUMO: Os homens se tornaram um desafio para as políticas públicas de saúde por representarem altos índices de mortalidade e morbidade, sendo as redes sociais uma das ferramentas para atrair a participação deles no cuidado com a sua saúde. O objetivo deste estudo foi analisar os meios de comunicação como estratégias de promoção da saúde do homem e de prevenção do câncer de próstata. Trata-se de um estudo descritivo, quantiqualitativo, realizado com 49 homens de uma universidade, no Município do Rio de Janeiro. A coleta de dados ocorreu através de questionário autoaplicável, em 2013. A análise de conteúdo dos depoimentos apontou as categorias - Os meios de comunicação, ferramentas da promoção da saúde e prevenção do câncer de próstata e a inclusão do homem na saúde preventiva. Conclui-se que os meios de comunicação tradicionais não surtiram os efeitos esperados. Os sujeitos utilizariam os meios alternativos de comunicação - como redes sociais, na tentativa de dar qualidade a sua saúde.
\end{abstract}

Palavras-Chave: Saúde do homem; meios de comunicação; neoplasias da próstata; promoção da saúde.

\begin{abstract}
Men have become a challenge for public health policies for showing high mortality and morbidity rates. Social networks have proven to be a useful tool to attract their participation in self-care. This study aimed at analyzing communication media strategies to men's health promotion and prevention of prostate cancer. This is a descriptive quantitative study, conducted with 49 men from a university in the city of Rio de Janeiro, RJ, Brazil. Data was collected through self-administered questionnaires in 2013 Content discourse analysis identified two categories: [1] media, tools for health promotion and prevention of prostate cancer; and [2] inclusion of men in preventive health. Conclusions show that traditional media have not achieved the expected results. Subjects would use alternative media, such as social networks, as an attempt to attain health quality. Keywords: Men's health; media; prostate neoplasms; health promotion.
\end{abstract}

RESUMEN: Los hombres se convirtieron en un desafío para las políticas de salud pública por representar una alta mortalidad y morbilidad, siendo las redes sociales una herramienta para atraer a su participación en el cuidado de su salud. El objetivo de este estudio fue analizar los medios de comunicación como estrategias para promover la salud de los hombres y prevenir el cáncer de próstata Se trata de un estudio descriptivo cuanticualitativo, hecho con, 49 hombres de una universidad en la ciudad de Río de Janeiro-Brasil. Los datos fueron recolectados a través de cuestionario autoaplicable, en 2013 El análisis de contenido de las declaraciones señaló las categorías - Los medios de comunicación, herramientas de promoción de la salud y prevención del cáncer de próstata; y la inclusión de los hombres en la salud preventiva. En conclusión, los medios tradicionales de comunicación no han logrado los resultados esperados. Los sujetos utilizarían medios alternativos - como las redes sociales, en un intento de dar calidad a su salud.

Palabras Clave: Salud del hombre; medios de comunicación; neoplasias de la próstata; promoción de la saúde.

\section{INTRODUÇÃO}

A presença masculina no cenário da saúde se tornou um grande desafio para as políticas públicas e os profissionais de saúde. Estudos mostram que homens representam índices de mortalidade e morbidade mais elevados do que as mulheres, evidenciando que a inclusão deles no serviço de atenção primária é menos expressiva do que a feminina ${ }^{1,2}$.

Uma das doenças com maior incidência em homens é o câncer de próstata. Esta é uma neoplasia de evolução lenta, de modo que a mortalidade poderia

${ }^{\mathrm{I} G r a d u a n d a}$ de Enfermagem da Universidade Veiga de Almeida. Rio de Janeiro, Brasil. E-mail: brunny.lima@gmail.com

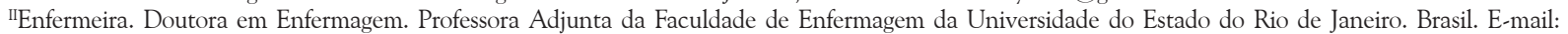
oigresrose@uol.com.br

IIIEnfermeira. Professora Substituta da Faculdade de Enfermagem da Universidade do Estado do Rio de Janeiro. Enfermeira da Policlínica Piquet Carneiro e da Urologia do Hospital Universitário Pedro Ernesto. Rio de Janeiro, Brasil. E-mail: raquel rcar@msn.com

${ }^{I V}$ Enfermeira. Doutoranda em Enfermagem. Professora Assistente da Faculdade de Enfermāem da Universidade do Estado do Rio de Janeiro. Chefe da Seção da Enfermaria de Urologia do Hospital Universitário Pedro Ernesto. Rio de Janeiro, Brasil. E-mail: cristiane.costa@ig.com.br

vEnfermeira. Professora Assistente da Faculdade de Enfermagem da Universidade do Estado do Rio de Janeiro. Brasil. E-mail: araciclos@yahoo.com.br

${ }^{\mathrm{VI} G r a d u a n d a ~ d e ~ e n f e r m a g e m ~ d a ~ U n i v e r s i d a d e ~ V e i g a ~ d e ~ A l m e i d a . ~ R i o ~ d e ~ J a n e i r o, ~ B r a s i l . ~ E-m a i l: ~ j a q u e l i n e l i t t r e l @ h o t m a i l . c o m ~}$ 
ser reduzida ou evitada com o diagnóstico precoce e a prevenção ${ }^{3}$. Existem diversos fatores que contribuem para a baixa inclusão deles na atenção primária, destacando-se a questão de gênero, cujas barreiras e aspectos simbólicos se antepõem à realização do exame de toque, que ainda é visto como invasivo e comprometedor da masculinidade ${ }^{3,4}$.

Além disso, o homem é marcado pelo estigma de cabeça da casa, sendo a ele atribuídas as responsabilidades econômicas, advindas do papel patriarcal. A demora do atendimento dos serviços de saúde, os horários que coincidem com o trabalho e a falta de tempo são argumentos utilizados para justificar sua reduzida participação no setor primário da saúde ${ }^{1}$.

Para maior adesão do gênero masculino aos serviços de saúde, seria necessário, maior oferta de meios de comunicação voltados para esta finalidade. Hoje, comerciais e informativos a respeito do cuidado com a saúde do homem são limitados. Estes meios deveriam chamar sua atenção para a promoção da saúde e prevenção de doenças e agravos, deixando-o à vontade e acolhido, assegurando seu espaço e suas condições de gênero.

As redes sociais podem ser uma das ferramentas para maior participação do gênero masculino. Um exemplo seria o movimento na internet chamado Movember-moustache (bigode) + november (novembro). Movimento, que nasceu na Austrália e já se espalhou pelo mundo através das redes sociais (facebook, twitter, blogs), traz campanhas de incentivo à prevenção do câncer de próstata de maneira diferente. Os participantes são desafiados a deixarem seus bigodes crescerem no mês de novembro como forma de lembrar e mostrar a importância de se realizar os exames para a detecção do câncer de próstata. Tal símbolo foi escolhido, segundo os organizadores, por sua marca genuína e representação do gênero masculino.

Diante da problemática apresentada, surge a seguinte questão norteadora do estudo: Qual é o impacto dessas ferramentas de comunicações alternativas - movimentos como Movember- na saúde do homem e na prevenção do câncer de próstata?

A realização deste estudo se justifica pela reduzida inclusão dos homens nos serviços de saúde da atenção primária, determinada principalmente por fatores culturais, como a forte questão de gênero,de onipotência, masculinidade e até mesmo o papel patriarcal e de provedor da família. Estes fatores contribuíram e, ainda, contribuem para os altos índices de morbidade e mortalidade do gênero masculino. Consequentemente, o número de doenças crônicas aumentou bruscamente no sexo masculino, principalmente o câncer de próstata.

Sendo assim, foi pensado como os meios de comunicação atuam para a promoção da saúde e prevenção do câncer de próstata a ponto de contribuir para a mudança desse quadro.
Os objetivos deste estudo foram: Analisar os meios de comunicação como estratégias de promoção da saúde do homem e prevenção de doenças como o câncer de próstata.

\section{REVISÃO DE LITERATURA}

A fim de promover maior inclusão do gênero masculino no setor de saúde, foi implantado, em 2008, no Brasil, a Política Nacional Integral à Saúde do Homem (PNAISH). Esta Política tem como objetivo qualificar a assistência à saúde do homem com uma linha de cuidado, de modo integral, na atenção primária. Pretende ir além da recuperação da saúde para garantir a prevenção de doenças e outros agravos e a promoção da saúde ${ }^{3-5}$.

Entre os princípios da PNAISH, ressaltam-se os educacionais, de modo que o homem se sinta acolhido e faça parte integrante do sistema de saúde. A educação seria uma porta de entrada juntamente com a atenção primária para o sistema de saúde universal, integral e equânime $e^{1-5}$.

\section{Metodologia}

Trata-se de um estudo com abordagem quantiqualitativa, que busca a explicação e a compreensão do fenômeno, descrevendo as variáveis e a dinâmica das relações sociais, em profundidade, além de trabalhar com valores, crenças e opiniões dos participantes ${ }^{6}$.

Participaram do estudo 49 homens, entre estudantes, docentes e trabalhadores de uma universidade localizada no Município do Rio de Janeiro. Os entrevistados situavam-se na faixa etária de 20 a 60 anos e foram escolhidos de forma aleatória. A partir da 49a entrevista, ocorreu a saturação dos dados - obtenção de respostas singulares - momento em que foi encerrada a coleta de dados.

O estudo foi aprovado pelo Comitê de Ética em Pesquisa da Universidade com número do CAAE: 700.005.291, sob o Parecer no 609.386.

Todos os participantes da pesquisa receberam o Termo de Consentimento Livre e Esclarecido (TCLE) e, após a assinatura do documento, foram entregues os questionários autoaplicáveis para aqueles que concordaram em respondê-los. Ressalta-se que foram garantidos o sigilo e anonimato dos participantes da pesquisa, de acordo com as exigências da Resolução n466/2012 do Conselho Nacional de Saúde ${ }^{7}$. Os sujeitos foram identificados pela letra $\mathrm{H}$ (homem) e o número sequencial de sua participação na entrevista.

Os dados foram coletados no período de setembro a dezembro de 2013, na referida universidade. Foi utilizado um questionário autoaplicável. A primeira parte compreendeu itens para a caracterização dos 
sujeitos do estudo, com destaque para a faixa etária e o número de vezes que procuraram ajuda de um profissional de saúde (enfermeiro, médico, ....) ou serviços de saúde.

Os dados coletados foram tratados segundo o método de análise de conteúdo. O objeto da análise de conteúdo é a palavra, aspecto individual da linguagem; seu objetivo são os significados dos vocábulos, tentando compreender os atores e o ambiente onde estão inseridos; é organizada em três etapas - pré-análise, exploração do material e tratamento dos resultados, inferência e interpretação ${ }^{8}$. Dessa análise, emergiram duas categorias que são tratadas na seção a seguir.

\section{Resultados e Discussão}

\section{Caracterização dos sujeitos}

Entre os 49 entrevistados, 20 eram estudantes, 15 docentes e 14 trabalhadores dos setores de infraestrutura da universidade, campo do estudo.

A maioria (43) situava-se na faixa etária de 20 a 30 anos, com representantes minoritários nas demais décadas, e a maioria (44) procurou a ajuda de um profissional de saúde, pelo menos uma vez, no ultimo ano.

A análise dos dados levou à construção de duas categorias: Os meios de comunicação, ferramentas na promoção da saúde e $\mathrm{A}$ inclusão do homem na saúde preventiva.

\section{Os meios de comunicação como ferramenta na promoção da saúde.}

Desta categoria emergiram as subcategorias Os meios de comunicação e Prevenindo o câncer de próstata.

\section{Os meios de comunicação}

Foi perguntado aos sujeitos se alguma campanha/ informativo e/ou veículo de comunicação (rádio, TV e internet) já os motivara a procurar os serviços de saúde, a maioria respondeu negativamente, porém os que foram motivados ressaltaram os aspectos negativos.

Sim, campanhas de imunização. A motivação vem das campanhas para evitar o adoecimento relacionado a doenças virais e bacterianas. (H21)

Já, tv, porque mostrou o que acontecia negativamente [caso não houvesse cuidados]. (H35)

Foi pela Internet, motivou devido a alterações cutâneas. (H48)

Sim, radio e tv, deixaram-me com a pulga atrás da orelha. (H36)

Pode-se perceber nos discursos apresentados que a motivação utilizada pelo meio de comunicação foi demonstrar o que acontecia negativamente em decorrência da não procura de assistência à saúde. Ressaltam-se as enfermidades como ponto mais abordado.

A prevenção de doenças, principalmente as bacterianas e virais, é um dos pontos abordados. Porém o que se mostra é que incentivos à própria promoção da saúde do homem ainda são escassos nas redes de comunicação. Assim, campanhas de mobilização nacional são as que chamam a atenção dele para a procura dos serviços.

A maioria afirmou que nenhuma campanha, informativo ou qualquer outro veículo o motivou.

Não. Nenhum me motivou. (H25)

Não. Mesmo nas situações, os veículos de comunicação não se mostram efetivos (H19)

A resistência dos homens em buscar os serviços de atenção básica, associada à falta de prevenção e autocuidado e à fragilidade e insegurança contrastam com a virilidade, exposição a situações de risco e invulnerabilidade, expressando traços culturais de uma visão hegemônica de masculinidade 9 .

Em relação aos meios de comunicação que poderiam abordar temas voltados para a saúde do homem, a maioria se referiu a propagandas, jornais, rádio e internet.

Em propagandas educativas e jornais $(\mathrm{H} 4)$

Com propagandas de horário nobre e divulgação de fotos no facebook. (H12)

Através de campanhas de tv relacionado à cerveja. (H15)

Por meio de propagandas em horário nobre, de pico de audiência à noite, e também por meio de rádios. (H21)

Um dos depoimentos menciona como os meios podem acrescentar aquilo que de fato pode chamar a atenção do homem, como a atração dos comerciais de cerveja.

Os profissionais de saúde devem considerar os homens como aliados, companheiros e detentores de uma herança cultural, oferecendo estratégias para desconstruir aspectos negativos da masculinidade e reconstruir ou enfatizar aspectos positivos, cujos valores são reforçados por fatores socioculturais e hábitos ${ }^{10}$.

\section{Prevenindo o câncer de próstata}

Quando perguntados se lembravam de algum veículo de comunicação, campanha e outros que abordassem o tema prevenção ao câncer de próstata, a maioria respondeu que não se lembrava.

Apesar de o estudo concentrar homens na faixa etária de 20 a 30 anos, é relevante que a conscientização e a informação sobre autocuidado cheguem cada vez mais cedo 1, $^{1,4,5}$. 
As respostas afirmativas mostram que a televisão ainda é um grande meio para se propagar e difundir a conscientização e a prevenção do câncer de próstata. Os homens lembraram de informativos e campanhas a respeito do câncer de próstata.

$$
\text { Sim. Na TV. (H45) }
$$$$
\text { Sim. Na TV. (H36) }
$$

\section{TV e Internet. (H7)}

Ao serem questionados se consideravam que os veículos de comunicação chamam a atenção para a saúde do homem e, principalmente, para a prevenção do câncer de próstata, a maioria negou a proposição.

A veiculação de campanhas sobre a prevenção do câncer de próstata é menor quando comparada ao câncer de mama e à saúde da mulher.

Nós vemos uma grande presença de informação quanto ao câncer de mama, porém nunca vi uma propaganda de informação sobre câncer de próstata. (H44)

Chamam a atenção de forma muito menor do que as campanhas de saúde da mulher. (H16)

A lógica dos serviços e a organização do cuidado em torno do eixo materno-infantil há anos acompanham o processo histórico que articulou a hegemonia das ideias médicas com as ações políticas voltadas para a valorização do corpo feminino ${ }^{11}$.

Na década de 80, com o desenvolvimento do Programa Política Assistencial de Saúde Integral à Mulher (PAISM), o panorama de atenção à saúde foi reorganizado por meio da articulação do referencial de gênero com o campo da saúde ao reafirmar a mulher como sujeito de direitos com necessidade de saúde e de individualidade própria ${ }^{12}$.

Desse modo, a maior presença feminina no setor da saúde também se dá pela apropriação do corpo feminino, corpo reprodutivo e de estratégias biomédicas. Já o corpo masculino restou como adjetivo reprodutor, um desconhecido e por referência a esse domínio da reprodução não foi investido como objetos de prática ${ }^{13}$.

Essa formulação é explicada pela abordagem homem e saúde ser mais recente sob a perspectiva dos profissionais de saúde e instituições. Além disso, a Política Nacional Integral à Saúde do Homem é recente, criada justamente pelos altos índices de morbidade e mortalidade do sexo masculino, inclusas as taxas de mortalidade e morbidade por câncer de próstata, que perdem apenas para o melanoma entre os homens ${ }^{3,13}$.

Vários fatores podem impedir a maior inclusão dos homens nos serviços de saúde, a predominância de mulheres nos espaços de atenção primária à saúde, sejam pacientes ou até profissionais de saúde e crianças; a eles sugerem um ambiente feminilizado ${ }^{14}$.

Nesse sentido, destacam-se as questões de vulnerabilidade acentuadas pelo gênero, é o caso do exame preventivo da próstata por meios do toque retal.
O toque retal é um exame de baixo custo e um efetivo detector do câncer de próstata, contudo é um procedimento que mobiliza o imaginário masculino, afastando inúmeros homens da prevenção. Este afastamento geralmente acontece pelas fortes tendências e raízes culturais marcantes do comportamento masculino ${ }^{15}$.

Quando perguntados, como as mídias tratam a questão do toque retal, a maioria respondeu que os meios de comunicação não tratam desta questão.

Na realidade não tratam e quando mencionam tratam de maneira dolorosa. (H49)

Não vejo divulgação, por isso acho que deveria ser melhor tratado. (H38)

Não falam nada.(H36)

Tratam? Nunca vi ou não me lembro de tratarem com finalidade educativa. Somente de forma pejorativa (H24)

Além dos depoimentos evidenciarem o silêncio sobre a questão do toque retal, o tema foi associado a piadas machistas. A maioria relatou que a mídia trata o exame de forma pejorativa e constrangedora.

Quase não tratam. Deixando com que haja espaço para certas brincadeiras, acabam faltando informações, impedindo que o indivíduo procure um médico para se cuidar. (H47)

Na maioria das vezes, como comédia. (H37)

Com preconceito. Principalmente em programas de comédia. (H14)

Como piada. (H27)

Tratam de maneira preconceituosa o tema, tornando alvo de shows de comédia ou situações cômicas. (H18)

Nota-se a escassez de veiculação a respeito do toque retal e mesmo quando é abordado, é de forma pejorativa e cômica. Ocasiona ainda mais o afastamento dos homens ao invés de um espaço aberto para que eles não se sintam constrangidos.

Exemplos de folders ou banners informativos das campanhas recentes de prevenção do câncer de próstata confirmam os discursos dos entrevistados certas mensagens de campanhas estampam palavras de duplo sentindo, levando para o lado cômico.

As palavras tocar e entrar estão muito presentes e de forma destacada nos informativos visuais para campanha do câncer de próstata. Isto gera um afastamento e constrangimento para o homem que lê esses informativos.

Além da televisão, as novas mídias, principalmente a internet, parecem ser um ótimo meio de propagação da informação e conscientização sobre a saúde do homem e, principalmente, a prevenção do câncer de próstata. Esses novos espaços aumentam a probabilidade de quebrar mitos e de propiciar formas alternativas de comunicação para o homem. Isto é 
confirmado quando os entrevistados mencionam que as formas alternativas de comunicação (facebook, twitter, orkut, blog, ...) são vantajosas para a divulgação da proteção da saúde masculina.

\section{A inclusão do homem na saúde preventiva}

Esta categoria clama pela inclusão do homem na saúde preventiva. Abrange as subcategorias Meios alternativos de comunicação e $\bigcirc$ homem participante dos meios de comunicação alternativos.

\section{Meios alternativos de comunicação}

Foi questionado aos participantes se tinham conhecimento a respeito do movimento na internet chamado Movember, que ganhou as redes sociais em todo mundo e que está fazendo grande sucesso no Brasil. A maioria afirmou não conhecer.

O movimento começou com a iniciativa de cinco amigos, Travis Garone, Lucas Slattery, Justin Coughlin e Adam Garone de Melbourne, na Austrália, em 2003, através de uma conversa sobre estilos e tendências desatualizadas, surgindo a ideia de trazer o bigode de volta. Logo aparece o nome do movimento-Movember (a combinação da gíria australiana para bigode, Mo e Novembro = november). As regras do movimento eram simples: começar em 1 de novembro com a barba feita e passar o restante do mês deixando o bigode crescer $^{16}$.

Vários fatores influenciaram a causa. $O$ primeiro foi inspirado pelo movimento mais conhecido na saúde da mulher, o câncer de mama. $\mathrm{O}$ segundo, quando perceberam que faltava uma maneira de envolver os homens nas suas próprias questões de saúde. Em 2004, o grupo aumentou para 450 participantes que abraçaram a causa, levantando recursos para as questões de saúde masculina principalmente o câncer de próstata. Foram arrecadados U\$ 55. 000 para a Fundação Câncer de Próstata da Austrália, a maior doação até então recebida ${ }^{16}$.

Assim o Movember veio crescendo gradativamente tornando o movimento global, inspirando mais de 1,1 milhões de participantes, todos conhecidos como Mo Bros e as mulheres, também encorajadas nesta causa, são conhecidas como Mo Sister. Além disso, o movimento é financiador de pesquisas como mapeamento genético do câncer de próstata (Programa de Sequenciamento do Genoma) e um que investiga a capacidade de nanoparticulas ligadas a anticorpos para melhorar a visualização na ressonância magnética em casos de câncer de próstata e aplicações de iluminação a lazer das nanoparticulas para permitir a visualização no intraoperatório de câncer de próstata ${ }^{16}$.

O que chama atenção desse movimento é a participação intensa dos homens. O principal veículo para essa disseminação é o boca a boca e as redes sociais. Logo se tornou uma das maiores forças organizada por homens, que traz em seu logo Changing the face of mens's health (Mudando a cara da saúde dos homens) na luta pela inclusão do homem na saúde e, consequentemente, contra o câncer de próstata ${ }^{17}$.

Pode-se notar que o movimento está inserido no Brasil de outras formas. No mundo da moda, o bigode está estampado em camisas, acessórios e bolsas, além do meio musical. A música bigode grosso, que foi hit de sucesso, traz um personagem que expõe uma atitude de respeito por seu bigode. Nesse aspecto, o bigode representa referencias de masculinidade, respeito e até virilidade, tornando-se um símbolo de identidade do gênero masculino.

\section{O homem participante dos meios de comunicação alternativos}

Quando indagados se fariam parte do movimento Movember, a maioria respondeu de maneira afirmativa. Esclareceram como podem participar do Movember de uma outra forma:

Desenhando um bigode e tirando uma foto para compartilhar nas redes sociais ( $\mathrm{H} 2)$

O uso da rede social como forma de divulgação. Seria interessante a criação de bigodes artificiais, caso algum homem não queira deixar o bigode crescer. (H15)

Mudando a foto do perfil do facebook,compartilhando fotos e acontecimentos reais e sociais [...]. (H21)

[...] espalharia a mensagem no facebook e etc. (H31)

Publicando em redes sociais. (H42)

As redes sociais virtuais oferecem a possibilidade de um debate aberto e plural, onde todos podem participar da criação e difusão da informação. Essas mídias demonstram ser uma plataforma ideal para a criação de eventos sociais, manifestos e campanhas virtuais ${ }^{18}$.

É importante dar voz aos homens, para que possam sugerir estratégias para o alcance maior dessa população. Para a mudança desse cenário, estratégias de ação dos profissionais de saúde devem ser revistas, de maneira a ampliar a participação dos homens nos serviços de saúde e no autocuidado, ajudando a reconhecer suas necessidades e a refletir acerca de seus comportamentos ${ }^{10}$.

A maioria dos entrevistados relatou que faria parte do movimento através dos meios alternativos de comunicação, (facebook,twitter, orkut e outros).

Outras formas de divulgação, como o compartilhamento e a difusão do movimento através do boca a boca e a inserção dos grupos sociais também são abordadas pelos sujeitos. São depoimentos:

Participaria, falando com todos que conheço sobre ele. (H5)

[...] participando, se pessoas do meu grupo social participassem.(H11)

Divulgando e fazendo educação em saúde. (H20) 
Outras maneiras de participar também foram apresentadas:

Usando uma camisa apoiando o movimento. (H4)

[...] colocação de um dia no calendário.

[...] usando um bigode postiço ou desenhando com maquiagem. (H10)

Colocar um tecido de determinada cor na janela. (H13)

Utilizaria uma pulseira ou cordão. (H24)

Deixando o cabelo crescer. (H28)

Usando camisas sobre... (H38)

[...] incentivar a adesão de formadores de opinião, como atores e atletas que difundiriam o movimento. (H44)

[...] mecha azul no cabelo. (H48)

Exposta a ideia do Movember aos entrevistados, foi perguntado se esses movimentos poderiam ser utilizados para maior conscientização de homens sobre o autocuidado; a maioria concordou que seriam eficazes para a promoção da saúde do homem e prevenção do câncer de próstata.

Em países como Canadá, Reino Unido, Estados Unidos e Austrália, percebeu-se uma forte adesão dos homens envolvidos nas causas da própria saúde e prevenção ao câncer de próstata, após alguns movimentos alternativos, os quais resultaram não só na maior participação em prol da saúde masculina, mas também na arrecadação de fundos para os projetos envolvidos nessas lutas ${ }^{16}$.

\section{CONClusão}

A maioria dos sujeitos pesquisados era jovem e buscou ajuda de profissional de saúde, pelo menos uma vez, no ultimo ano.

$\mathrm{Na}$ categoria Os meios de comunicação como ferramenta na promoção da saúde, foi destacado que a maioria não incentiva o homem a procurar o serviço de saúde e, quando o faz, principalmente através da televisão, não é de maneira construtiva e há menor ênfase, em comparação às campanhas de saúde da mulher.

Na segunda categoria Inclusão do homem na saúde preventiva, há um clamor pela adesão masculina ao autocuidado e às formas alternativas de divulgação.

Percebe-se que os meios de comunicação atuais não estão apresentando resultados efetivos para a superação dos desafios das Políticas Públicas de Saúde para a inclusão dos homens na atenção básica. São escassos os recursos, propagandas, informativos voltados para esta população, e quando aparecem não agradam o público masculino, além de ainda se constatar uma atenção à saúde privilegiada para o público materno-infantil.
Vale registrar a importância do tema, principalmente, pelos agravos à saúde e altos índices de morbidade e mortalidade masculina, entre os quais o câncer de próstata.

As redes sociais como meios de divulgação para a saúde do homem e prevenção do câncer de próstata são valiosas, tendo em visto, as declarações dos sujeitos pesquisados.

É importante que a enfermagem participe dessas ações, nos meios de comunicação alternativos, como as redes sociais, incentivando as práticas de autocuidado para o alcance de melhor qualidade de vida e mudanças da face da saúde dos homens.

Entre as limitações do estudo, destacam-se a reduzida amostra e um único cenário que impedem a generalização dos achados.

\section{REFERÊNCIAS}

1.Gomes R, Nascimento EF, Araújo, FC. Por que os homens buscam menos os serviços de saúde do que as mulheres? As explicações de homens com baixa escolaridade e homens com ensino superior. Cad Saúde Pública. 2009; 23:565-74.

2.Brito RS, Santos DLA. Entraves para a implementação de programas assistenciais dirigidos ao público masculino: visão de profissionais de saúde. Rev enferm UERJ. 2013; 21:654-9. 3.Ministério da Saúde (Br). Política Nacional Integral à Saúde do Homem. Brasilia (DF): Editora MS; 2008. 4.Silva ABM, Costa, CMA, Spíndola T, Ramos RCA, Martins ERC, Francisco MTR. Conhecimentos e práticas sobre prevenção do câncer de próstata: uma contribuição para a enfermagem. Rev enferm UERJ. 2013; 21:785-91. 5.Gomes R, Nascimento EF, Rebello LEFS, Araújo FC. As arranhaduras da masculinidade: uma discussão sobre o toque retal como medida de prevenção ao câncer prostático. Ciência e Saúde Coletiva. 2008; 13: 1975-84. 6.Lakatos EM, Marconi MA. Fundamentos de metodologia científica. 3a ed. São Paulo: Atlas; 1991.

7.Ministério da Saúde (Br). Resolução nº 466/12 do Conselho Nacional de Saúde. Diretrizes e Normas Regulamentadoras de pesquisa envolvendo seres humanos. Brasília (DF): CNS; 2012

8.Bardin L. Análise de conteúdo. Lisboa $(\mathrm{Pt})$ : Edições 70; 2011.

9.Schwarz E. Reflexões sobre o gênero e a Política Nacional de Atenção Integral à Saúde do Homem. Ciência e Saúde Coletiva. 2012; 17: 2579-88.

10.Paschoalik RC, Lacertda MR, Centa ML. Gênero masculino e saúde. Cogitare Enfermagem. [ periódico na Internet] 2006. [citado em 12 abr 2014]; 11(4) [ aprox. 7telas]. Disponível em: http://ojs.c3sl.ufpr.br/ojs/index. $\mathrm{php} /$ cogitare/article/view/5979

11.Costa AM. Desenvolvimento e implantação do PAISM no Brasil: questões de saúde reprodutiva. Rio de Janeiro: Editora FIOCRUZ; 1999.

12.Villella W, Monteiro S. Atenção à saúde das mulheres: historicizando conceitos e práticas. In: Villela W, Monteiro 
S, organizadores. Gênero e saúde: programa saúde da família em questão. Rio de Janeiro: Abrasco; 2005. p. 15-32.

13.Intituto Nacional do Câncer (Br). Câncer de próstata.2014. [citado em 14 abr 2014]. Disponível em: http:// www2.inca.gov.br/wps/wcm/connect/tiposdecancer/site/ home/prostata]

14.Figueiredo W. Assistência à saúde dos homens. Ciência \& Saúde Coletiva [online].2005 [citado em 12 abr 2014] 10(1) [aprox. 5 telas]. Disponível em http://www.scielo.br/ 15.Gomes R. Sexualidade masculina e saúde do homem: proposta para discussão. Ciência $\&$ Saúde Coletiva [online].2003. [citado em 12 abr 2014] 8(3): [aprox. 5 telas]. Disponível em http://www.scielo.br/

16.Jeffcoff M, Cagiannos L, Zorn K. Movember update:
The Canadian perspective. US Nacional Library of Medicine Nacional Institutes of health. Can Urol Assoc J. 2012 [cited in 2014 Apr 14]; 6(3): E111-4. Disposable in: http:/www.ncbi.nlm.nih.gov/pmc/articles/pmc3377735/ 17.Siemens R. Connecting with prostate cancer survivors. US Nacional Library of Medicine Nacional Institutes of Health. Can Urol Assoc J. 2012 [cited in 2014 Apr 14]; 6(5): 349. Disposable in: http://www.ncbi.nlm. nih.gov/pmc/articles/PMC3478331/

18.Cardoso G, Lamy C. Redes sociais e mudança na comunicação. E- journal of Internacional Relations [Universidade Autônoma de Lisboa] 2011; [citado em 14 abr 2014] 2(1):73-96. Disponível em: dialnet.unirioja. es/descarga/articulo/4046121/3.pdf 